Pacific Journal of Mathematic 


\title{
A “GOING DOWN" THEOREM FOR CERTAIN REFLECTED RADICALS
}

\author{
B. J. GARDNER AND PATrick N. StewART
}

\begin{abstract}
In a category $\mathscr{K}$ suitable for radical theory, a functor $\Phi: \mathscr{K} \rightarrow \mathscr{K}$ is studied which is associated with a natural transformation $1_{\mathscr{r}} \rightarrow \Phi$ in a way which bears a formal resemblance to the behavior of certain "extension" functors of rings, such as that which assigns to each $A$ the polynomial ring $A[x]$ : every normal subobject $N \rightarrow \Phi(A)$ has a "contraction" $N^{c} \rightarrow A$. For a radical class $\mathscr{R}$ in $\mathscr{C}$ such that $\mathscr{R}^{*}=$ $\{A \mid \Phi(A) \in \mathscr{R}\}$ is also radical, some conditions are obtained which imply that $\mathscr{R} *(A)=\mathscr{R}(\Phi(A))^{c}$.
\end{abstract}

1. Preliminaries. We shall work in a category $\mathscr{K}$ for which the general theory of radicals can be developed (for a set of conditions on $\mathscr{K}$ which ensure this and for some other remarks on radicals in categories, see [9]) and shall consider a left-exact functor $\Phi: \mathscr{C} \rightarrow$ $\mathscr{C}$ which has associated with it a natural transformation $1_{\mathscr{C}} \rightarrow \Phi$, which will be fixed throughout the discussion. We shall further assume that for each normal subobject $N \rightarrow \Phi(A)$ there is a normal subobject $N^{c A} \rightarrow A$ and a pullback

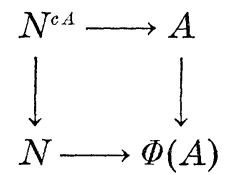

where the right-hand vertical map is defined by the natural transformation mentioned above. When no confusion can result, $N^{c A}$ will be abbreviated to $N^{c}$. We shall frequently find it convenient to write $A^{e}$ for $\Phi(A)$. A prototypical example of such a functor is that which assigns to each ring $A$ its polynomial ring $A[x]$, in which case $A^{e}=$ $A[x]$ ("extension") and $N^{c}=N \cap A$ ("contraction"). The symbol $A \rightarrow A^{e}$ will always denote a map defined by the given natural transformation.

Our category-theoretic terminology is essentially that of [2]. We shall not distinguish notationally between a subobject and a representative map. In particular if $A \in \mathscr{K}$ and $\mathscr{R}$ is a radical class, $\mathscr{R}(A) \rightarrow$ $A$ will denote the $\mathscr{R}$-radical of $A$.

Proposition 1.1 .

(a) If $N \rightarrow A$ is a normal subobject, then $N \rightarrow A \subseteq N^{e c} \rightarrow A$.

(b) If $N_{1} \rightarrow A^{e} \subseteq N_{2} \rightarrow A^{e}$ are normal subobjects then $N_{1}^{c} \rightarrow A \subseteq$ $N_{2}^{c} \rightarrow A$. 
(c) $A^{e c}=A$.

(d) If $I \rightarrow A$ and $J \rightarrow A^{e}$ are normal subobjects, with $J \rightarrow A^{e} \subseteq$ $I^{e} \rightarrow A^{e}$, then there is a map $J^{c I} \rightarrow J^{c A}$ such that

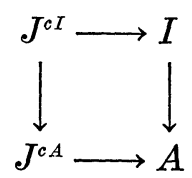

commutes.

(e) If

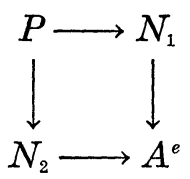

is a pullback and $N_{1} \rightarrow A^{e}, N_{2} \rightarrow A^{e}, P \rightarrow A^{e}$ are normal subobjects, then

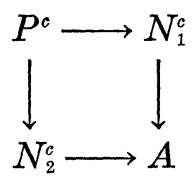

is also a pullback.

Proof. (a) follows from consideration of the diagram

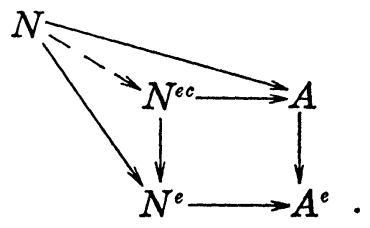

(b) follows from consideration of

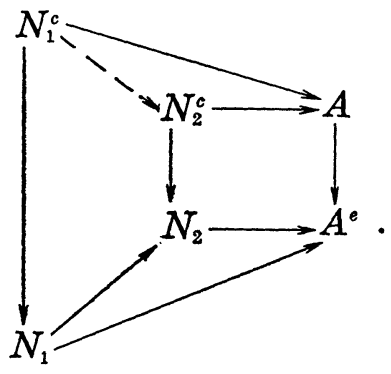

(c) is obtained from (a) by taking $N=A$. 
A “GOING DOWN" THEOREM FOR CERTAIN REFLECTED RADICALS 383

(d) Since $I^{e} \longrightarrow A^{e}, J^{c I} \longrightarrow I$

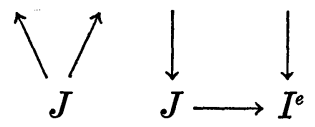

and $J^{c A} \longrightarrow A$ commute and the last square is a pullback, consideration<smiles>C[14CH][Y][Te]C</smiles>

of the following diagram establishes the result.

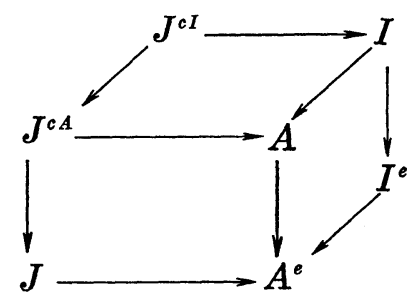

(e) Consider the diagram

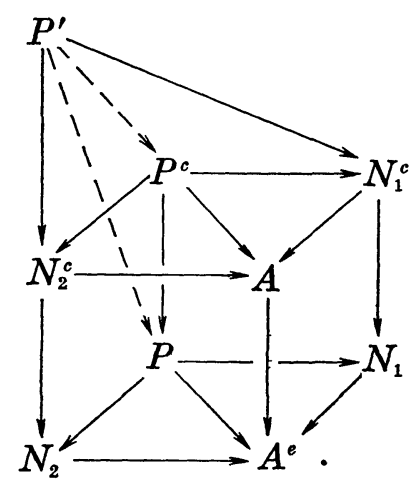

By (b) the two triangles on the top of the cube commute and so the square on the top of the cube commutes. Suppose

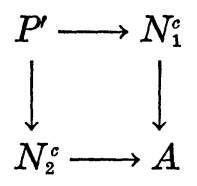

commutes. Since the base of the cube is a pullback we obtain an appropriate map $P^{\prime} \longrightarrow P$ and since

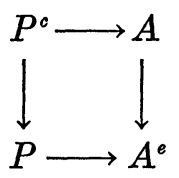


is a pullback, we obtain the required map $P^{\prime} \rightarrow P^{c}$ which is unique since $P^{c} \rightarrow A$ is monic.

2. The results. If $\mathscr{R}$ is a radical class in $\mathscr{K}$, we denote by $\mathscr{R}^{*}$ the class $\{A \in \mathscr{K} \mid \Phi(A) \in \mathscr{R}\}$. Henceforth we shall only consider radical classes $\mathscr{R}$ for which $\mathscr{R}^{*}$ is also a radical class. Some conditions on $\Phi$ which imply that $\mathscr{R}^{*}$ is radical for some or all radical classes $\mathscr{R}$ are given in [5].

Proposition 2.1. For every $A \in \mathscr{K}$, we have $\mathscr{R}^{*}(A) \rightarrow A \subseteq$ $\mathscr{R}\left(A^{e}\right)^{c} \rightarrow A$.

Proof. Since $\mathscr{R}^{*}(A)^{e} \rightarrow A^{e}$ is a normal $\mathscr{R}$-subobject we have $\mathscr{R}^{*}(A)^{e} \rightarrow A^{e} \subseteq \mathscr{R}\left(A^{e}\right) \rightarrow A^{e}$. The result now follows from (a) and (b) of Proposition 1.1.

A radical class $\mathscr{R}$ is hereditary if $N \in \mathscr{R}$ whenever $M \in \mathscr{R}$ and $N \rightarrow M$ is a normal subobject. $\mathscr{R}$ is normally-hereditary if for every normal subobject $N \rightarrow A$ we have

$$
\mathscr{R}(N) \longrightarrow N \longrightarrow A=(N \rightarrow A) \cap(\mathscr{R}(A) \longrightarrow A) \text {. }
$$

Clearly normally-hereditary radical classes are hereditary, but the converse need not be true.

In what follows we shall be concerned with the following conditions involving $\Phi$ and $\mathscr{R}$.

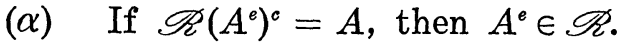

$\left(\alpha^{\prime}\right) \quad \mathscr{R}\left(A^{e}\right)^{c e} \rightarrow A^{e} \subseteq \mathscr{R}\left(A^{e}\right) \rightarrow A^{e}$ for each $A \in \mathscr{K}$.

$\left(\alpha^{\prime \prime}\right) \quad \mathscr{R}\left(A^{e}\right)^{c e} \in \mathscr{R}$ for each $A \in \mathscr{K}$.

It is easy to see that $\left(\alpha^{\prime \prime}\right) \Rightarrow\left(\alpha^{\prime}\right) \Rightarrow(\alpha)$.

PROPOSITION 2.2. If every pair of normal subobjects of each object in $\mathscr{K}$ has a normal intersection, then for normally-hereditary radical classes $\mathscr{R},(\alpha)$ and $\left(\alpha^{\prime}\right)$ are equivalent.

Proof. We need only show that $(\alpha)$ implies $\left(\alpha^{\prime}\right)$. Assume $(\alpha)$ is satisfied. The pullback

$(*)$

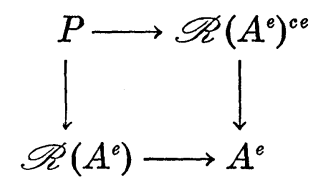

exists. By Proposition 1.1(e) 


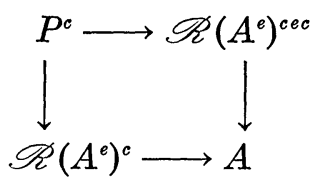

is a pullback and by Proposition 1.1(a), $\mathscr{R}\left(A^{e}\right)^{c} \rightarrow A \subseteq \mathscr{R}\left(A^{e}\right)^{c c c} \rightarrow A$ and so $P^{c}=\mathscr{R}\left(A^{e}\right)^{c}$. Since $\mathscr{R}$ is normally-hereditary, it follows from

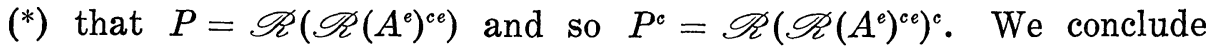
that $\mathscr{R}\left(A^{e}\right)^{c}=\mathscr{R}\left(\mathscr{R}\left(A^{e}\right)^{c e}\right)^{c}$, i.e., $\mathscr{R}\left(I^{e}\right)^{c A}=I$, where $I=\mathscr{R}\left(A^{e}\right)^{c}$.

Consider the diagram

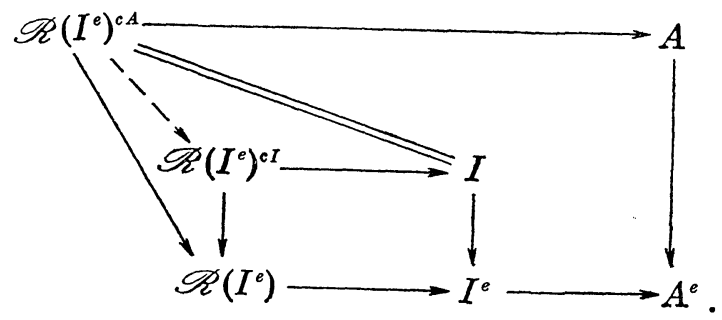

Since $I^{e} \rightarrow A^{e}$ is monic and

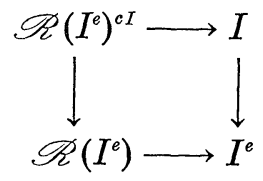

is a pullback we obtain a map $\mathscr{R}\left(I^{e}\right)^{c A} \rightarrow \mathscr{R}\left(I^{e}\right)^{c I}$ such that

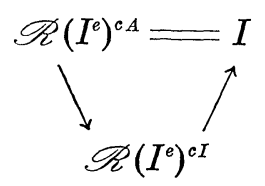

commutes. Thus $\mathscr{R}\left(I^{e}\right)^{c I}=I$ and so by $(\alpha)$ we have $\mathscr{R}\left(A^{e}\right)^{c e}=I^{e} \epsilon$ $\mathscr{R}$, which establishes $\left(\alpha^{\prime}\right)$.

Proposition 2.3. If $\mathscr{R}$ is hereditary, then $\left(\alpha^{\prime}\right)$ and $\left(\alpha^{\prime \prime}\right)$ are equivalent.

Proof. Obvious.

Proposition 2.4. If $\mathscr{R}$ satisfies $(\alpha)$ then

$$
\mathscr{R}^{*}=\left\{A \mid \mathscr{R}\left(A^{e}\right)^{c}=A\right\} \text {. }
$$

Proof. If $\mathscr{R}\left(A^{e}\right)^{c}=A$, then $A^{e} \in \mathscr{R}$, i.e., $A \in \mathscr{R}^{*}$, by $(\alpha)$. Conversely, if $A \in \mathscr{R}^{*}$, then $A^{e} \in \mathscr{R}$ and so $\mathscr{R}\left(A^{e}\right)=A^{e}$. Hence $\mathscr{R}\left(A^{e}\right)^{c}=$ $A^{e c}=A$ by Proposition 1.1(c). 
Proposition 2.4 gives a "global" description of $\mathscr{R}^{*}$. We can also give a "local" description of $\mathscr{R}^{*}$ under more restrictive conditions. We shall need

Lemma 2.5. If $\mathscr{R}$ satisfies $\left(\alpha^{\prime \prime}\right)$ then for each $A \in \mathscr{K}$ we have

$$
\mathscr{R}\left(A^{e}\right)^{c e c}=\mathscr{R}\left(A^{e}\right)^{c} .
$$

Proof. By $\left(\alpha^{\prime \prime}\right), \mathscr{R}\left(A^{e}\right)^{c e} \in \mathscr{R}$, so

$$
\mathscr{R}\left(\mathscr{R}\left(A^{e}\right)^{c e}\right)^{c}=\mathscr{R}\left(A^{e}\right)^{c e c} \text { and } \mathscr{R}\left(A^{e}\right)^{c e} \longrightarrow A^{e} \subseteq \mathscr{R}\left(A^{e}\right) \longrightarrow A^{e} \text {. }
$$

The latter implies, by Proposition 1.1(b), that $\mathscr{R}\left(A^{e}\right)^{c e c} \rightarrow A \subseteq \mathscr{R}\left(A^{e}\right)^{c} \rightarrow$ $A$ and from Proposition 1.1(a) we see that $\mathscr{R}\left(A^{e}\right)^{c} \rightarrow A \subseteq \mathscr{R}\left(A^{e}\right)^{c e c} \rightarrow A$.

THeOREM 2.6. If $\mathscr{R}$ satisfies $(\alpha)$ and $\mathscr{R}\left(\mathscr{R}\left(A^{e}\right)^{c e}\right)^{c}=\mathscr{R}\left(A^{e}\right)^{c}$, then $\mathscr{R}^{*}(A)=\mathscr{R}\left(A^{e}\right)^{c}$.

Proof. Applying Proposition 2.4 to $\mathscr{R}\left(A^{e}\right)^{c}$, we see that $\mathscr{R}\left(A^{e}\right)^{c} \in$ $\mathscr{R}^{*}$. The equality now follows from Proposition 2.1.

COROLLARY 2.7. If every pair of normal subobjects of each object of $\mathscr{K}$ has a normal intersection and if $\mathscr{R}$ is normally-hereditary and satisfies $(\alpha)$, then $\mathscr{R}^{*}(A)=\mathscr{R}\left(A^{e}\right)^{c}$ for each $A \in \mathscr{K}$.

Proof. By Proposition 2.2, $\mathscr{R}$ satisfies $\left(\alpha^{\prime}\right)$ and hence, by Proposition 2.3, $\left(\alpha^{\prime \prime}\right)$. By Lemma 2.5, $\mathscr{R}$ satisfies the requirements of Theorem 2.6 for all $A \in \mathscr{K}$.

3. Examples. In the category of associative rings, the functor $\Phi$ which associates the semigroup ring $A[S]$ with a ring $A(S$ is a fixed semigroup with identity) and acts on maps in the obvious way satisfies the requirements listed in $\S 1$, the natural transformation being defined by the standard embedding $A \rightarrow A[S]$. Moreover, $\mathscr{R}^{*}=\{A \mid A[S] \in \mathscr{R}\}$ is radical for every radical class $\mathscr{R}$ (cf. [5]) and $\mathscr{R}^{*} \subseteq \mathscr{R}$.

The following result is essentially due to Krempa [7] who proved it in the special case where $S$ is the free semigroup with identity on one generator, i.e., $A[S]$ is the polynomial ring $A[x]$.

Proposition 3.1. Every radical class of associative rings satisfies $\left(\alpha^{\prime}\right)$ for the functor defined by the correspondence $A \mapsto A[S]$, for any semigroup $S$.

From Proposition 2.4 we see that $\mathscr{R}^{*}=\{A \mid \mathscr{R}(A[S]) \cap A=A\}$ for every radical class $\mathscr{R}$. Thus in the case $A^{e}=A[x], \mathscr{R}^{*}$ coincides with the radical class discussed by Ortiz [8]. 
Proposition 2.1 and Theorem 2.6 therefore generalize Theorem 1 of [8]. By Corollary 2.7, $\mathscr{R}^{*}(A)=\mathscr{R}(A[S]) \cap A$ whenever $\mathscr{R}$ is hereditary. For $A[S]=A[x]$, this was proved by the first author in [4].

Another example of a functor defined on the category of associative rings which meets our requirements is that which assigns to each ring $A$ the ring $A_{n}$ of $n \times n$ matrices for some (fixed) $n$. Again action on maps is defined in the obvious way. The natural transformation is defined by the embedding of $A$ in $A_{n}$ as the subring of scalar matrices. In this case too, $\mathscr{R}^{*}=\left\{A \mid A_{n} \in \mathscr{R}\right\}$ is radical for all radical classes $\mathscr{R}$ [5].

The proof of the following result closely resembles that of Proposition 3.1 .

Proposition 3.2. Every radical class $\mathscr{R}$ of associative rings satisfies $\left(\alpha^{\prime}\right)$ for the functor defined by the correspondence $A \mapsto A_{n}$.

By Proposition 2.4, $\mathscr{R}^{*}=\left\{A \mid A \subseteq \mathscr{R}\left(A_{n}\right)\right\}$ in this case and by Corollary 2.7, $\mathscr{R}^{*}(A)=\mathscr{R}\left(A_{n}\right) \cap A$ when $\mathscr{R}$ is hereditary. (Here we have identified $A$ with the ring of scalar matrices.)

Let $0 \rightarrow Z \rightarrow X \rightarrow D \rightarrow 0$ be an exact sequence of abelian groups, where $Z$ is the group of integers and $D$ is torsion-free divisible. The functor ()$\otimes X$ has a right adjoint and so $\mathscr{R}^{*}=\{G \mid G \otimes X \in \mathscr{R}\}$ is a radical class for every radical class $\mathscr{R}$ of abelian groups [5]. The map $G \rightarrow G \otimes X$ defined by the isomorphism $G \cong G \otimes Z$ and the given exact sequence defines a natural transformation from the identity to $(\quad) \otimes X$. All requirements of $\S 1$ are satisfied.

Proposition 3.5. Every radical class $\mathscr{R}$ of abelian groups satisfies $(\alpha)$ for the functor ()$\otimes X$.

Proof. If $G \subseteq \mathscr{R}(G \otimes X)$, there is an epimorphism

$$
G \otimes D \cong(G \otimes X) / G \longrightarrow(G \otimes X) / \mathscr{R}(G \otimes X) .
$$

If $\mathscr{R}$ contains only torsion groups, then $G$ is torsion and so $G \otimes D=$ $0 \in \mathscr{R}$. If $\mathscr{R}$ contains a nontorsion group, then it contains all divisible groups (see e.g. [3], Corollary 2.3) and so $G \otimes D \in \mathscr{R}$. Hence $(G \otimes X) / \mathscr{R}(G \otimes X) \in \mathscr{R}$ in all cases. Thus $G \otimes X \in \mathscr{R}$.

In all the examples considered so far, the natural transformation involved has arisen from a natural embedding $A \rightarrow A^{e}$. We conclude with a simple example in which the relevant map $A \rightarrow A^{e}$ need not be monic.

Let $R_{1}$ and $R_{2}$ be associative rings with identity, $R=R_{1} \oplus R_{2}$ 
(ring direct sum) and let $\Phi$ be the functor defined on the category Mod $(R)$ of right unital $R$-modules by $M \mapsto M R_{1}$. The classes $\mathscr{R}_{i}=$ $\left\{M R_{i} \mid M \in \operatorname{Mod}(R)\right\}, i=1,2$, are actually hereditary radical classes and we have the situation analysed in Theorem 2.4 of Jans [6]. It is straightforward to show that $\Phi$ is exact and preserves unions of ascending chains and hence (see [5]) that $\mathscr{R}^{*}=\{M \mid \Phi(M) \in \mathscr{R}\}$ is a radical class for every radical class $\mathscr{R}$ in $\operatorname{Mod}(R)$. The projection $M \rightarrow M R_{1}$ defines a natural transformation with the properties we want. If $N$ is a submodule of $M R_{1}=M^{e}$ then $N^{c}=N \oplus M R_{2}$.

Proposition 3.3. Every radical class $\mathscr{R}$ in $\operatorname{Mod}(R)$ satisfies $(\alpha)$ for the functor defined by the correspondence $M \mapsto M R_{1}$.

Proof. If $\mathscr{R}\left(M R_{1}\right) \oplus M R_{2}=M=M R_{1} \oplus M R_{2}$, then $M R_{1}=$ $\mathscr{R}\left(M R_{1}\right)$.

4. The question of "going up". We revert to our general situation to briefly mention a related problem: to determine when $\mathscr{R}\left(A^{e}\right)=\mathscr{R}^{*}(A)^{e}$. Since $\mathscr{R}^{*}(A) \in \mathscr{R}^{*}$, we always have $\mathscr{R}^{*}(A)^{e} \rightarrow$ $A^{e} \subseteq \mathscr{R}\left(A^{e}\right) \rightarrow A^{e}$. The other inclusion seems to be more difficult. Amitsur [1] has given a (highly nontrivial) proof for $A^{e}=A[x]$ which is valid when $\mathscr{R}$ is strongly hereditary or the Jacobson radical class. On the other hand, when $A^{e}=A_{n}$, it is relatively easy to check that $\mathscr{R}\left(A^{e}\right)=\mathscr{R}^{*}(A)^{e}$ for all rings $A$ when $\mathscr{R}$ is hereditary.

Proposition 4.1. Let $A$ be an associative ring, $\mathscr{R}$ a radical class of associative rings. If $A$ has an identity or $\mathscr{R}$ is hereditary, then $\mathscr{R}\left(A_{n}\right)=\mathscr{R}^{*}(A)_{n}$.

Proof. If $A$ has an identity, then $\mathscr{R}\left(A_{n}\right)=I_{n}$ for some ideal $I$ of $A$. Since $I_{n} \in \mathscr{R}$ we have $I \in \mathscr{R}^{*}$; thus $I \subseteq \mathscr{R}^{*}(A)$ and so $\mathscr{R}\left(A_{n}\right) \subseteq$ $\mathscr{R}^{*}(A)_{n}$. But $\mathscr{R}^{*}(A)_{n} \in \mathscr{R}$, so $\mathscr{R}\left(A_{n}\right)=\mathscr{R}(A)_{n}$. If $A$ does not have an identity and $\mathscr{R}$ is hereditary, we make use of the Dorroh extension $A^{1}$ of $A$. Because $\mathscr{R}$ is hereditary (= normally-hereditary), so is $\mathscr{R}^{*}$, and thus we have $\mathscr{R}\left(A_{n}\right)=A_{n} \cap \mathscr{R}\left(\left(A^{1}\right)_{n}\right)=A_{n} \cap \mathscr{R}^{*}\left(A^{1}\right)_{n}=$ $\left[A \cap \mathscr{R}^{*}\left(A^{1}\right)\right]_{n}=\mathscr{R}^{*}(A)_{n}$.

\section{REFERENCES}

1. S. A. Amitsur, Radicals of polynomial rings, Canad. J. Math., 8 (1956), 355-361.

2. P. Freyd, Abelian Categories, Harper and Row, 1964.

3. B. J. Gardner, Two notes on radicals of abelian groups, Comment. Math. Univ. Carolinae, 13 (1972), 419-430.

4. — A note on radicals and polynomial rings, Math. Scand., 31 (1972), 8388. 
5. B. J. Gardner and P. N. Stewart, Reflected radical classes, (in preparation).

6. J. P. Jans, Some aspects of torsion, Pacific J. Math., 15 (1965), 1249-1259.

7. J. Krempa, On radical properties of polynomial rings, Bull. Acad, Polon. Sci. Sér. Sci. Math. Astronom. Phys., 20 (1972), 545-548.

8. A. H. Ortiz, A construction in general radical theory, Canad. J. Math., 22 (1970), 1097-1100.

9. E. G. Šul'geifer, On the general theory of radicals in categories, Amer. Math. Soc. Transl. (2) 59, 150-162. Russian original: Mat. Sb. 51 (1960), 487-500.

Received December 4, 1973.

University of Tasmania, Hobart, Tasmania, Australia

AND

Dalhousie University, Halifax, Nova Scotia, Canada 



\section{PACIFIC JOURNAL OF MATHEMATICS}

\section{EDITORS}

RICHARD ARENS (Managing Editor)

University of California

Los Angeles, California 90024

\section{R. A. Beaumont \\ University of Washington \\ Seattle, Washington 98105}

\section{J. DugundJI}

Department of Mathematics

University of Southern California

Los Angeles, California 90007

D. Gilbarg and J. Milgram

Stanford University

Stanford, California 94305

\section{ASSOCIATE EDITORS}
E. F. BECKENBACH
B. H. NeumanN
F. WOLF
K. YOSHIDA

\section{SUPPORTING INSTITUTIONS}

UNIVERSITY OF BRITISH COLUMBIA
CALIFORNIA INSTITUTE OF TECHNOLOGY
UNIVERSITY OF CALIFORNIA
MONTANA STATE UNIVERSITY
UNIVERSITY OF NEVADA
NEW MEXICO STATE UNIVERSITY
OREGON STATE UNIVERSITY
UNIVERSITY OF OREGON
OSAKA UNIVERSITY

UNIVERSITY OF BRITISH COLUMBIA

UNIVERSITY OF CALIFORNIA

MONTANA STATE UNIVERSITY

NEW MEXICO STATE UNIVERSITY

OREGON STATE UNIVERSITY

OSAKA UNIVERSITY

\author{
UNIVERSITY OF SOUTHERN CALIFORNIA \\ STANFORD UNIVERSITY \\ UNIVERSITY OF TOKYO \\ UNIVERSITY OF UTAH \\ WASHINGTON STATE UNIVERSITY \\ UNIVERSITY OF WASHINGTON \\ AMERICAN MATHEMATICAL SOCIETY \\ NAVAL WEAPONS CENTER
}

The Supporting Institutions listed above contribute to the cost of publication of this Journal, but they are not owners or publishers and have no responsibility for its content or policies.

Mathematical papers intended for publication in the Pacific Journal of Mathematics should be in typed form or offset-reproduced, (not dittoed), double spaced with large margins. Underline Greek letters in red, German in green, and script in blue. The first paragraph or two must be capable of being used separately as a synopsis of the entire paper. Items of the bibliography should not be cited there unless absolutely necessary, in which case they must be identified by author and Journal, rather than by item number. Manuscripts, in triplicate, may be sent to any one of the editors. Please classify according to the scheme of Math. Reviews, Index to Vol. 39. All other communications should be addressed to the managing editor, or Elaine Barth, University of California, Los Angeles, California, 90024.

The Pacific Journal of Mathematics expects the author's institution to pay page charges, and reserves the right to delay publication for nonpayment of charges in case of financial emergency.

100 reprints are provided free for each article, only if page charges have been substantially paid. Additional copies may be obtained at cost in multiples of 50 .

The Pacific Journal of Mathematics is issued monthly as of January 1966. Regular subscription rate: $\$ 72.00$ a year (6 Vols., 12 issues). Special rate: $\$ 36.00$ a year to individual members of supporting institutions.

Subscriptions, orders for back numbers, and changes of address should be sent to Pacific Journal of Mathematics, 103 Highland Boulevard, Berkeley, California, 94708.

PUBLISHED BY PACIFIC JOURNAL OF MATHEMATICS, A NON-PROFIT CORPORATION

Printed at Kokusai Bunken Insatsusha (International Academic Printing Co., Ltd.), 270, 3-chome Totsuka-cho, Shinjuku-ku, Tokyo 160, Japan.

Copyright (C) 1973 by Pacific Journal of Mathematics Manufactured and first issued in Japan 


\section{Pacific Journal of Mathematics \\ Vol. 55, No. $2 \quad$ October, 1974}

Walter Allegretto, On the equivalence of two types of oscillation for elliptic

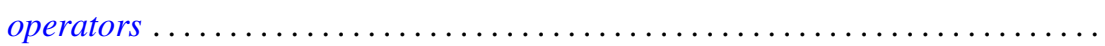

Edward Arthur Bertram, A density theorem on the number of conjugacy classes in

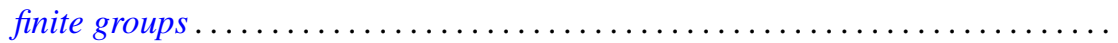

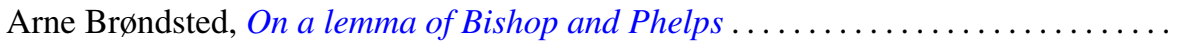

Jacob Burbea, Total positivity and reproducing kernels ..................

Ed Dubinsky, Linear Pincherle sequences . . . . . . . . . . . . . . . . . .

Benny Dan Evans, Cyclic amalgamations of residually finite groups .............

361

Barry J. Gardner and Patrick Noble Stewart, A "going down" theorem for certain

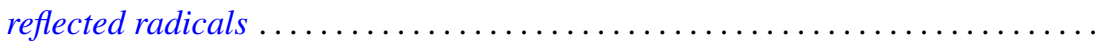

381

Jonathan Light Gross and Thomas William Tucker, Quotients of complete graphs:

revisiting the Heawood map-coloring problem ....................

Sav Roman Harasymiv, Groups of matrices acting on distribution spaces .........

Robert Winship Heath and David John Lutzer, Dugundji extension theorems for

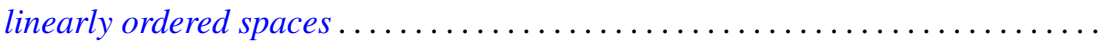

Chung-Wu Ho, Deforming p. l. homeomorphisms on a convex polygonal

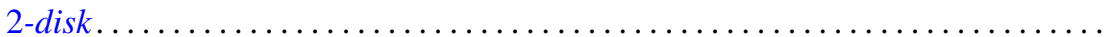

Richard Earl Hodel, Metrizability of topological spaces .................

Wilfried Imrich and Mark E. Watkins, On graphical regular representations of

cyclic extensions of groups .......................... 461

Jozef Krasinkiewicz, Remark on mappings not raising dimension of curves ..... . 479

Melven Robert Krom, Infinite games and special Baire space extensions . . . . . . 483

S. Leela, Stability of measure differential equations . . . . . . . . . . . . . . . . 489

M. H. Lim, Linear transformations on symmetric spaces . . . . . . . . . . . . . . . 499

Teng-Sun Liu, Arnoud C. M. van Rooij and Ju-Kwei Wang, On some group algebra modules related to Wiener's algebra $M_{1} \ldots \ldots \ldots \ldots \ldots \ldots \ldots \ldots \ldots \ldots \ldots$

Dale Wayne Myers, The back-and-forth isomorphism construction ............ 521

Donovan Harold Van Osdol, Extensions of sheaves of commutative algebras by

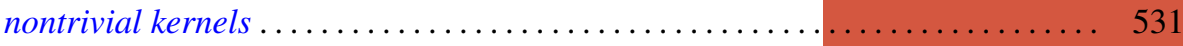

Alan Rahilly, Generalized Hall planes of even order ................... 543

Joylyn Newberry Reed, On completeness and semicompleteness of first countable

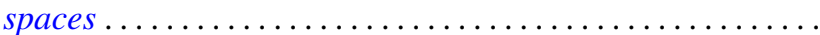

Alan Schwartz, Generalized convolutions and positive definite functions associated

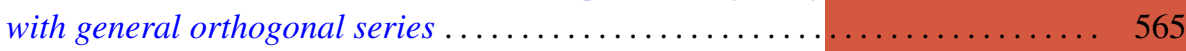

Thomas Jerome Scott, Monotonic permutations of chains . . . . . . . . . . . 583

Eivind Stensholt, An application of Steinberg's construction of twisted groups .... 595

Yasuji Takeuchi, On strongly radicial extensions . . . . ................. 619

William P. Ziemer, Some remarks on harmonic measure in space . . . . . . . . . . 629

John Grant, Corrections to: “Automorphisms definable by formulas” . . . . . . . . 639

Peter Michael Rosenthal, Corrections to: "On an inversion for the general

Mehler-Fock transform pair" ......................... 640

Carl Clifton Faith, Corrections to: "When are proper cyclics injective” . . . . . . 640 